\title{
Construction of a Biosensor Operating on the Combined Principles of Electrochemical Analysis and Localized Surface Plasmon Resonance for Multiple Detection of Antigen-Antibody and Enzymatic Reactions on the Single Biosensor
}

\author{
Tatsuro Endo ${ }^{1,2, *}$, Hikaru Takizawa1 ${ }^{1}$ Yasuko Yanagida ${ }^{1,2}$, \\ Takeshi Hatsuzawa ${ }^{1,2}$ and Eiichi Tamiya ${ }^{3}$ \\ ${ }^{1}$ Department of Mechano-Micro Engineering, Interdisciplinary Graduate School \\ of Science and Engineering, Tokyo Institute of Technology, \\ 4259 Nagatsuta-cho, Midori-ku, Yokohama 226-8502, Japan \\ ${ }^{2}$ Precision and Intelligence Laboratory, Tokyo Institute of Technology, \\ 4259 Nagatsuta-cho, Midori-ku, Yokohama 226-8503, Japan \\ ${ }^{3}$ Department of Applied Physics, Graduate School of Engineering, Osaka University, \\ 2-1 Yamadaoka, Suita, Osaka 565-0871, Japan
}

(Received April 22, 2008; accepted September 2, 2008)

Key words: biosensor, localized surface plasmon resonance (LSPR), electrochemical analysis, hemoglobin A1c (HbA1c)

In this paper, we describe the construction of a biosensor operating on the combined principles of electrochemical analysis and localized surface plasmon resonance (E-LSPR) using a core-shell nanoparticle-layer substrate for medical applications. The biosensor thus constructed enabled the multiple detection of antigen-antibody and enzymatic reactions on the single biosensor. In addition, we used the biosensor to detect glucose and hemoglobin A1c (HbA1c) for application in diabetes diagnostics. Antigen-antibody reactions were detected on the basis of the principle of LSPR-based optical detection, and enzymatic reactions were detected on the basis of the principle of electrochemical detection using the single biosensor. The E-LSPR biosensor was characterized on the basis of these optical and electrochemical detection principles and the biosensor construction procedure. We found that this biosensor could be used to specifically detect glucose and HbA1c and that the detection of these target molecules using this biosensor was simpler, requiring smaller sample volumes than those required by conventional biosensors. Thus, the E-LSPR biosensor has potential for use in cost-effective, simple, and highly sensitive test kits for medical applications.

*Corresponding author: e-mail: endo.t.ab@m.titech.ac.jp 


\section{Introduction}

The use of biosensors in medical diagnostics enables the establishment of simpler and more cost-effective instrumentation. ${ }^{(1)}$ Furthermore, biosensors are expected to serve as highly sensitive, high-throughput on-site monitoring systems that have a considerably wider range of functions than conventional bioassays such as the enzyme-linked immunosorbent assay (ELISA) and the polymerase chain reaction (PCR). Conventional bioassays have the following disadvantages: they present difficulties with regard to liquid handling, are time consuming, and require expensive desktop apparatus. To overcome these limitations, several detection principles and construction designs of biosensors have been studied. ${ }^{(2,3)}$ In particular, biosensors used in medical fields, i.e., miniaturized biosensors based on the micro-electro-mechanical systems (MEMS) technology, have facilitated a remarkable breakthrough with regard to the quantitative determination of target molecules. The MEMS technology has enabled the construction of micrometersized biosensors that are gradually gaining widespread acceptance. ${ }^{(4,5)}$

Biosensors that can be used as diagnostic tools for diabetes have been intensively investigated. ${ }^{(6,7)}$ Since diabetic patients need to regularly monitor their blood glucose levels, MEMS-based biosensors are extremely useful for off-site monitoring by these patients. In addition, biosensors that operate on different detection principles such as optical and electrochemical detection have been developed, and several MEMS-based biosensors have been commercialized for diabetes diagnostics. However, there remains a demand for more sensitive, cost-effective, and simpler biosensors. For the construction of a biosensor that fulfills all the above-mentioned requirements, we focused on the specific characteristics of nanomaterials.

Nanotechnology involving the use of nanomaterials such as nanotubes, nanoparticles, and nanofibers is a novel and interesting field of research. In this regard, the inherently small size and unique optical, magnetic, catalytic, and mechanical properties of nanomaterials, which are not displayed by bulk materials, have been theoretically and experimentally studied in detail. In addition, these specific characteristics of nanomaterials have been actively exploited in electronic, optical, and biotechnological applications. ${ }^{(8-10)}$ Furthermore, the nanoparticles and nanostructures of noble metals such as gold and silver have become the focus of researchers in many fields over the past decade because of their interesting properties and applications in biotechnology, surface science, optics, and electronics. ${ }^{(11,12)}$ This has led to many theoretical and experimental studies on the properties of noble-metal nanoparticles. In particular, the application of these nanoparticles in chemical sensors and biosensors has attracted attention. Noblemetal nanoparticles are sensitive to changes in the surrounding environment, such as in the refractive indices of different media. Furthermore, they can be specifically observed in the visible region (380-750 nm) of absorption spectra owing to their property of localized surface plasmon resonance (LSPR) absorption. These features enable the establishment of a simple and cost-effective optical experimental setup. On the basis of these specific optical characteristics and experimentally determined features of noblemetal nanoparticles and nanostructures, many researchers have investigated their use in sensing applications. ${ }^{(13,14)}$ However, the previously reported LSPR-based chemical 
sensors and biosensors required the synthesis of the noble-metal nanoparticles for sensor fabrication. Moreover, sophisticated techniques are required to obtain highly uniform noble-metal nanoparticles and nanostructures.

We have previously achieved the label-free detection of antigen-antibody reactions, cell metabolites, and peptide nucleic acid (PNA)-DNA and DNA-DNA hybridizations using an LSPR-based optical biosensor having a core-shell nanoparticle layer as the

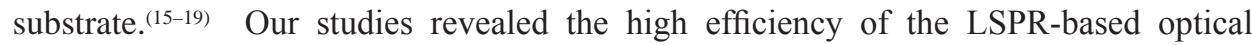
biosensor. In addition, by exploiting the characteristics of this biosensor, we were able to fabricate a multiarray LSPR-based optical biosensor for use as a protein array biosensor. $^{(17)}$ The core-shell nanoparticle-layer substrate for LSPR excitation was constructed using a surface-modified silica nanoparticle as the "core" and a gold layer as the "shell;" the shell was thermally deposited onto the core surface. Thus, the substrate for LSPR excitation was fabricated without the use of sophisticated techniques such as those required for noble-metal nanoparticle synthesis. In addition, we found that the substrate for LSPR excitation could be used as an electrode. ${ }^{(20)}$ Therefore, in this study, we aimed to develop a biosensor operating on the combined principles of electrochemical analysis and LSPR (E-LSPR) for use in diabetes diagnostics using a core-shell nanoparticle layer as the substrate. Using this construction, the multiple detection principles can be applied to the single biosensor. Therefore, using this biosensor for the medical applications, the detailed clinical conditions of patients can be determined. Furthermore, using this biosensor, the experimental instrumentation can be simplified.

As reported previously, commercially available biosensors have been used for diabetes diagnostics; these biosensors are used to detect blood glucose levels by detecting enzymatic reactions, operating mainly on the principle of electrochemical detection. ${ }^{(21,22)}$ However, blood glucose levels continuously fluctuate and therefore require regular monitoring. Hence, the use of conventional biosensors for blood glucose monitoring in diabetes diagnostics is both physically and mentally stressful. To overcome these limitations of conventional biosensors with regard to diabetes diagnostics, we aimed to detect hemoglobin $(\mathrm{Hb}) \mathrm{A} 1 \mathrm{c}$ using an E-LSPR biosensor. Using our single biosensor for the detection of glucose and HbA1c, the physical conditions of diabetic patients can be clarified in more detail.

A wide variety of parameters such as glucose, HbA1c, insulin, and glycated albumin (GA) can be quantified for the diagnosis of diabetes. The standard values of these parameters and the techniques used to detect them, e.g., enzymatic assay, ELISA, and chemiluminescent enzyme immunoassay (CLEIA), differ. Of these parameters, HbA1c has recently attracted attention since it has great potential for use in diabetes diagnostics. $\mathrm{Hb}$ is the oxygen-carrying pigment responsible for the red color of blood and is the predominant protein in red blood cells (RBCs). In addition, HbA accounts for approximately $90 \% \mathrm{Hb}$. Although $92 \% \mathrm{HbA}$ is composed of a single component, approximately $8 \%$ is composed of minor components, whose chemical compositions differ to some extent. These minor components include HbA1c, HbA1b, HbA1a1, and HbA1a2. Among these, HbA1c is the $\mathrm{Hb}$ component to which glucose binds, and its level depends on the concentration of glucose in the blood. However, the HbA1c level is not affected by daily fluctuations in the blood glucose level but reflect the average 
glucose level over the previous 6-8 weeks. Therefore, the HbA1c level is a useful indicator of how well the blood glucose level has been controlled in the recent past, and it may be used to monitor the effects of diet, exercise, and drug therapy on the blood glucose levels of diabetic patients. High-performance liquid chromatography (HPLC) and/or latex agglutination (LA) are typically used to determine the HbA1c level. These detection methods are disadvantageous in that they are time-consuming, present difficulties with regard to liquid handling, and require large sample volumes and expensive instruments.

In this study, considering the above-mentioned properties of $\mathrm{HbA} 1 \mathrm{c}$ as a marker for diabetes diagnostics, we attempted to detect glucose and HbA1c using an E-LSPR biosensor. We fabricated an E-LSPR biosensor and characterized it using a UV-VIS spectrophotometer and a potentiostat. Furthermore, the glucose and HbA1c levels were determined through the detection of enzymatic and antigen-antibody reactions, respectively, using an E-LSPR biosensor.

\section{Materials and Methods}

\subsection{Materials}

To generate the nanoparticle layer, 4,4'-dithiodibutyric acid (DDA) purchased from Aldrich was used for the formation of a self-assembled monolayer (SAM) on the gold substrate surface. 1-Ethyl-3-(3-dimethylaminopropyl) carbodiimide (EDC), used for the activation of the carboxyl group of DDA, was purchased from Dojindo Laboratories (Kumamoto, Japan). 3-Aminopropyltriethoxysilane ( $\gamma$-APTES), used for the surface modification of silica nanoparticles, was purchased from Shin-Etsu Chemical Co., Ltd. (Tokyo, Japan). Silica nanoparticles (100 nm i.d.), used for preparing the nanoparticle layer, were purchased from Polysciences Inc. (Warrington, PA). The glass substrate (10 $\times 26 \mathrm{~mm}$; thickness, $1.1 \mathrm{~mm}$ ) was purchased from Fujimoto Science Co., Ltd. (Tokyo, Japan). $N$-hydroxysuccinimide (NHS) for antibody immobilization and for the reaction with a glucose oxidase (GOx) (EC 1.1.3.4; extracted from Aspergillus niger; 20,000 units/g solid) was purchased from Wako Pure Chemical Industries, Ltd. (Osaka, Japan). The human anti-HbA1c monoclonal antibody and human HbA1c were purchased from Cosmo Bio (Tokyo, Japan). Ultrapure water $(18.3 \mathrm{M} \Omega-\mathrm{cm})$ procured from Millipore was used in all the experiments.

\subsection{Apparatus}

For the deposition of gold and titanium onto the glass substrate, a sputtering apparatus (L250S-FH) was purchased from Anelva Co., Ltd. (Kanagawa, Japan). A spectrophotometer (USB 4000 UV-VIS; wavelength range, 200-1100 nm), a tungsten halogen light source (LS-1; wavelength range, 360-2000 nm), and an optical-fiber probe bundle (R-200-7 UV/VIS; fiber core diameter, $200 \mu \mathrm{m}$; wavelength range, 250-800 nm), used to evaluate the optical characteristics of the E-LSPR biosensor, were purchased from Ocean Optics (Dunedin, USA). To perform electrochemical analysis using the E-LSPR biosensor, the microAutolab III system ( $\mu$ AUT III) was purchased from Eco Chemie (Utrecht, Netherlands). 


\subsection{Fabrication of the E-LSPR biosensor using a core-shell nanoparticle-layer substrate}

The biosensor was fabricated using a core-shell nanoparticle-layer substrate by the following method. First, 1 mM DDA solution was applied onto the titanium- (5 nm) and gold-coated $(40 \mathrm{~nm})$ glass substrate, whose surface was modified using the sputtering apparatus; the SAM was thus generated within $1 \mathrm{~h}$. The SAM was rendered functional by treating it with $400 \mathrm{mM}$ EDC solution for $1 \mathrm{~h}$; EDC activated the carboxyl groups of DDA, and the amino groups of the surface-modified silica nanoparticles formed esters with the activated carboxyl groups. The surface-modified silica nanoparticles were further modified with amino groups by treatment with a silane-coupling reagent ( $\gamma$-APTES) in ultrapure water and were exposed to the activated SAM-modified glass substrate surface for $1 \mathrm{~h}$. The nanoparticle-layer substrates were thoroughly rinsed with ultrapure water to remove the excess surface-modified nanoparticles and were dried at room temperature (RT). Finally, a gold layer (30 nm) was deposited on the nanoparticlelayer substrates using the sputtering apparatus. Following this fabrication procedure, the color generated due to LSPR absorption was monitored in the visible region. Thus, the E-LSPR biosensor was constructed (Fig. 1). In addition, to perform electrochemical analysis using the E-LSPR biosensor, the two-electrode method was applied in this study. Working and counter electrodes were fabricated onto the glass substrate by micromachining. The nanoparticle layer was then generated on the surface of the working electrode, which had been fabricated using the procedure described above.

\subsection{Experimental setup and detection principles of E-LSPR biosensor}

The experimental setup of the E-LSPR biosensor is schematically illustrated in Fig. 2. In this study, the LSPR-based optical detection principle was applied to detect the antigen-antibody reactions, and the electrochemical detection principle was applied to detect the enzymatic reactions. This experimental setup of the E-LSPR biosensor enabled the simultaneous detection of antigen-antibody and enzymatic reactions.

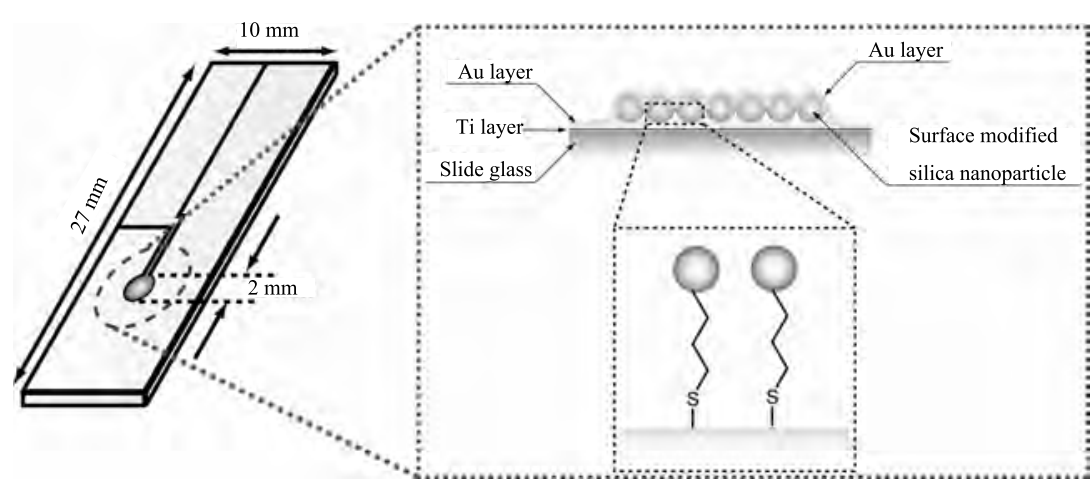

(a)

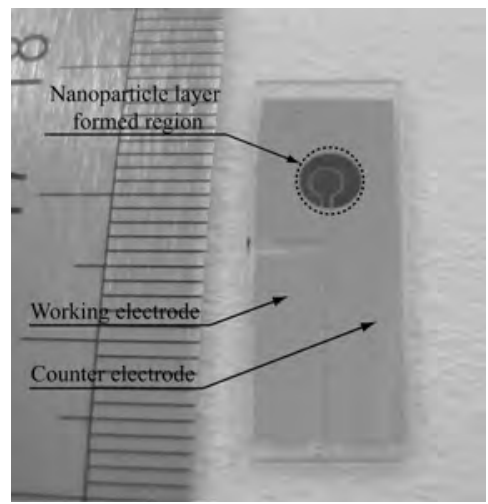

(b)

Fig. 1. (a) Construction of the E-LSPR biosensor. (b) Photograph of the E-LSPR biosensor. 


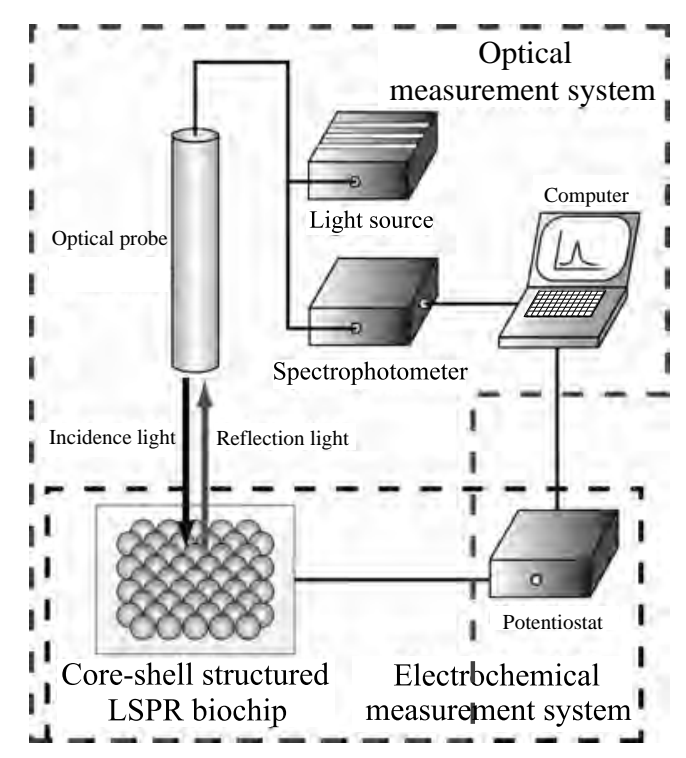

Fig. 2. Schematic illustration of the experimental setup of the E-LSPR biosensor.

The principle by which the E-LSPR biosensor could detect antigen-antibody reactions, which was based on the LSPR-based optical detection principle, is schematically illustrated in Fig. 3. For the detection of the HbA1c antigen, the antiHbA1c antibody was immobilized onto the E-LSPR biosensor surface, and then the sample antigen solution was introduced onto the E-LSPR biosensor surface. The introduction of the antigen solution altered the refractive index of the biosensor surface due to specific antigen-antibody interactions that occurred in an antigen-concentrationdependent manner $(1 \mathrm{ng} / \mathrm{ml}-100 \mu \mathrm{g} / \mathrm{ml})$. Consequently, the LSPR optical characteristics of the biosensor surface were altered. For antibody immobilization, $10 \mu \mathrm{g} / \mathrm{ml}$ of the antibody solution was introduced onto the E-LSPR biosensor surface; the antibodies were immobilized onto the surface via physical adsorption. Excess antibodies were removed by washing with phosphate buffer $(20 \mathrm{mM}, \mathrm{pH}$ 7.4). Thus, the E-LSPR biosensor carrying immobilized antibodies was obtained.

The principle by which the E-LSPR biosensor could detect enzymatic reactions, which was based on electrochemical detection, is schematically illustrated in Fig. 4. For the detection of glucose concentration, the hydrogen peroxide released during enzymatic reactions was quantified using chronoamperometry. First, $5 \mu \mathrm{l}$ of $\mathrm{GOx}(10 \mathrm{U} / \mathrm{ml})$ and $15 \mu \mathrm{l}$ of glucose $(0-10 \mathrm{mM})$ were mixed, and the resultant solution was introduced onto the surface of the electrodes. Thereafter, electrochemical analysis was performed. In addition, the chronoamperometry involved hydrogen peroxide oxidation by the application of a $0.8 \mathrm{~V}$ potential for $120 \mathrm{~s}$ in phosphate buffer. The glucose-concentrationdependent oxidation currents produced under these experimental conditions were monitored. 

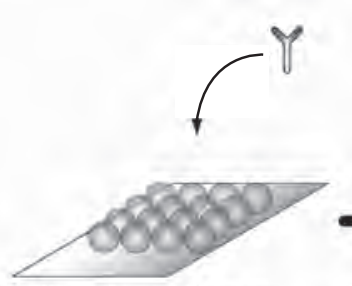

1) Antibody immobilization

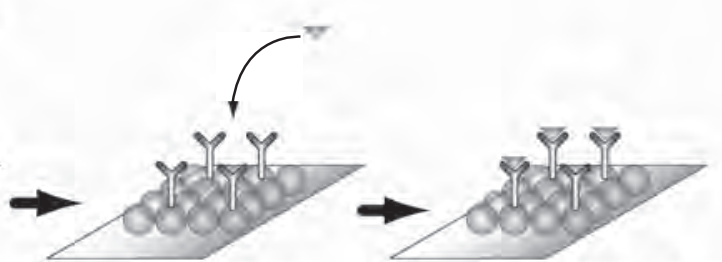

2) Antigen-antibody reaction

3) Optical characteristics evaluation

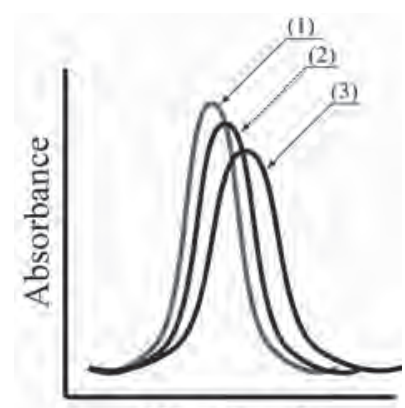

Wavelength

Fig. 3. LSPR-based optical detection principle.

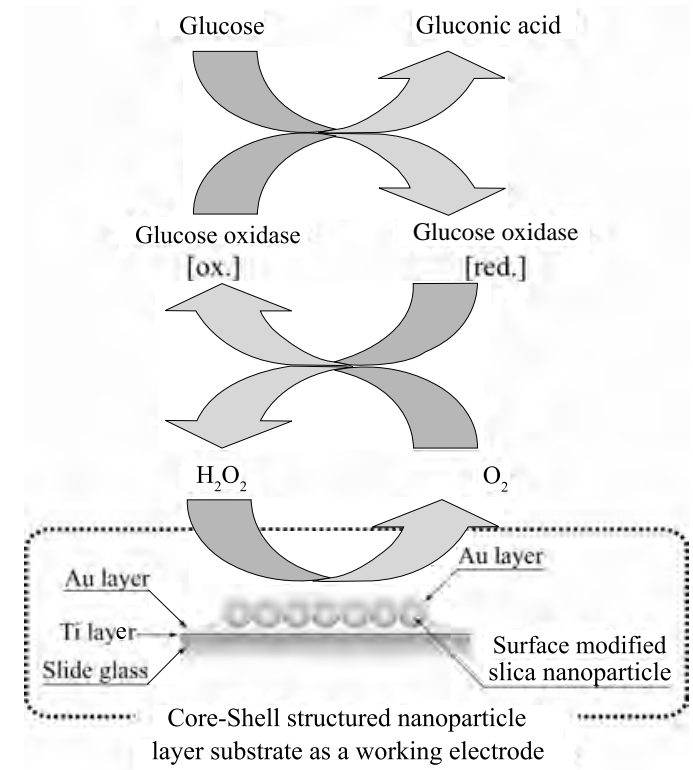

Fig. 4. Electrochemical detection principle of the E-LSPR biosensor.

\section{Results and Discussion}

\subsection{Characteristics of the E-LSPR biosensor}

The LSPR optical characteristics of the E-LSPR biosensor are shown in Fig. 5. After the E-LSPR biosensor was fabricated as described above, the LSPR absorbance peak was observed at $533 \mathrm{~nm}$. Antigen-antibody reactions were detected on the basis of these LSPR optical characteristics of the E-LSPR biosensor.

The cyclic voltammogram obtained for the E-LSPR biosensor in phosphate buffer is shown in Fig. 6. Cyclic voltammetry (potential, $0-800 \mathrm{mV}$; scan rate, $50 \mathrm{mV} / \mathrm{s}$ ) was 

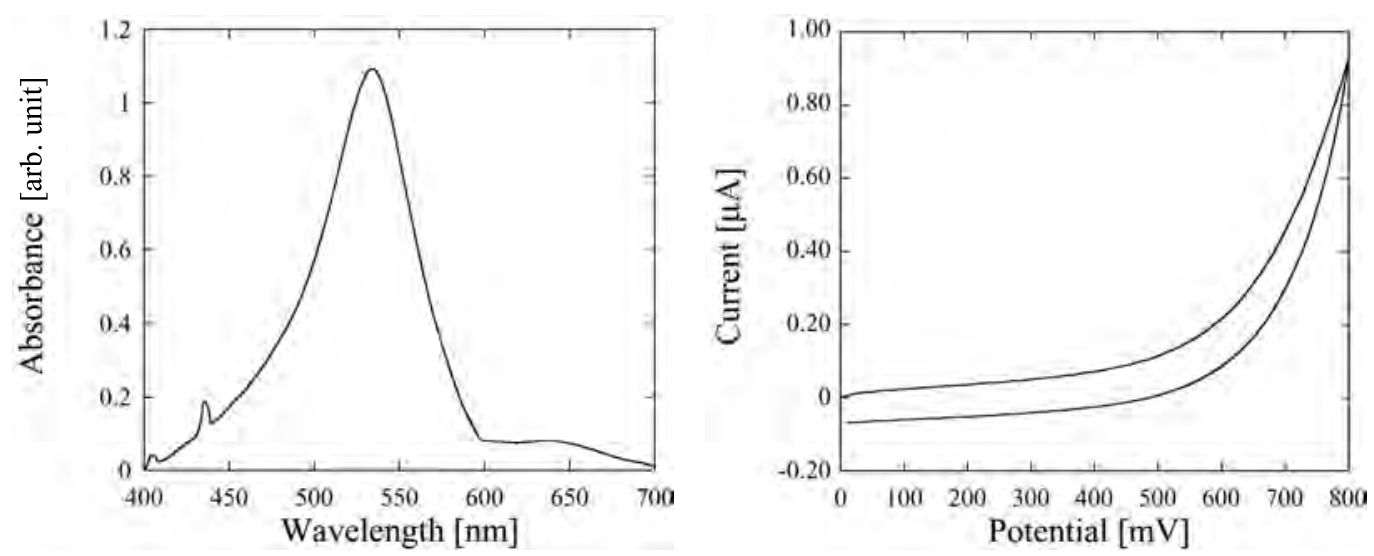

Fig. 5 (left). LSPR optical characteristics of the E-LSPR biosensor.

Fig. 6 (right). Electrochemical characteristics of the E-LSPR biosensor.

performed to evaluate the electrochemical analysis ability of the biosensor. From the electrochemical characteristic of our E-LSPR biosensor, it was found that the E-LSPR biosensor can be used in electrochemical analyses. Therefore, it was used to determine blood glucose concentrations on the basis of the principle of electrochemical detection.

\subsection{HbA1c detection using the E-LSPR biosensor based on the LSPR-based optical detection principle}

To quantify HbA1c, the antigen-antibody reaction was detected using the E-LSPR biosensor. The changes in the LSPR optical characteristics induced by the antigenantibody reactions are shown in Fig. 7. We observed a change in the LSPR absorbance strength and a shift in the LSPR peak wavelength. These changes in the optical characteristics were attributed to the change in the refractive index of the biosensor surface that was induced by specific antigen-antibody interactions. However, the HbA1c-concentration-dependent variations in the antigen-antibody reactions could not be observed using the E-LSPR biosensor. This defective quantitative capability is due to the low immobilization efficiency of the antibody onto the E-LSPR biosensor surface. In this study, the antibody was immobilized onto the E-LSPR biosensor surface by physical adsorption. Hence, it became extremely difficult to preserve the stable antibody conformation while maintaining the adsorption efficiency. Thus, the detection limit of this biosensor for the quantitative determination of HbAlc was insufficient to satisfy the criteria for diabetes diagnostics based on blood parameters. Therefore, further investigations are required to optimize the procedure for constructing the E-LSPR biosensor. 


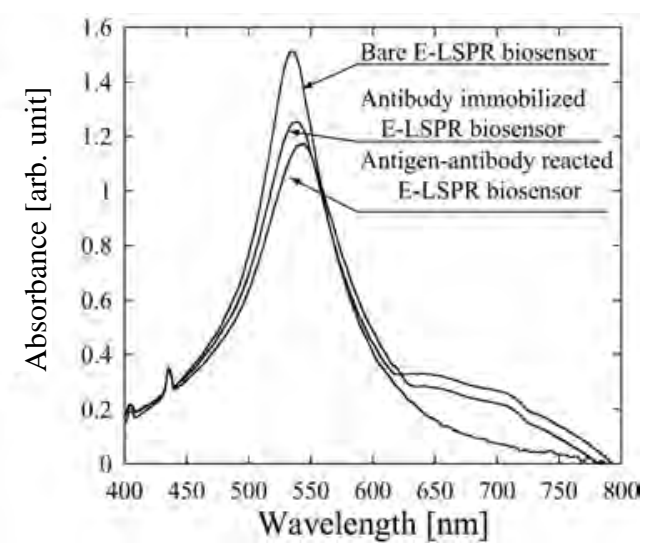

Fig. 7. Changes in the LSPR optical characteristics of the E-LSPR biosensor due to antigenantibody reactions.

\subsection{Quantitative determination of glucose concentrations using the E-LSPR biosensor based on the electrochemical detection principle}

The calibration characteristics for glucose detection using the E-LSPR biosensor are shown in Fig. 8. In this figure, the sensor output is presented as a changing output current value. The blood glucose levels in healthy subjects remain within a narrow range throughout the day $(4-8 \mathrm{mM})$. Therefore, in this study, glucose solutions at concentrations of $0-10 \mathrm{mM}$ were used for the evaluation of the calibration characteristics based on the principle of electrochemical detection. As shown in Fig. 8, the output current of the E-LSPR biosensor is linearly related to the glucose concentration in the range of $0-10 \mathrm{mM}$; the correlation coefficient was determined to be 0.988 by performing regression analysis.

For further evaluation of the calibration characteristics, an enzyme-glucose mixture was introduced onto the E-LSPR biosensor surface. Initially, an E-LSPR biosensor carrying only the immobilized enzyme was used to detect the glucose concentrations. The enzyme was immobilized onto the biosensor surface by physical adsorption. However, glucose-concentration-dependent variations in the enzyme reactions (data not shown) could not be determined using this biosensor. Therefore, the enzyme-glucose mixture was introduced onto the biosensor surface.

Furthermore, in this experiment, the two-electrode system was applied for the detection of glucose using the electrochemical detection principle. Hence, this biosensor needs to be improved to reduce the interferences and current drift. 


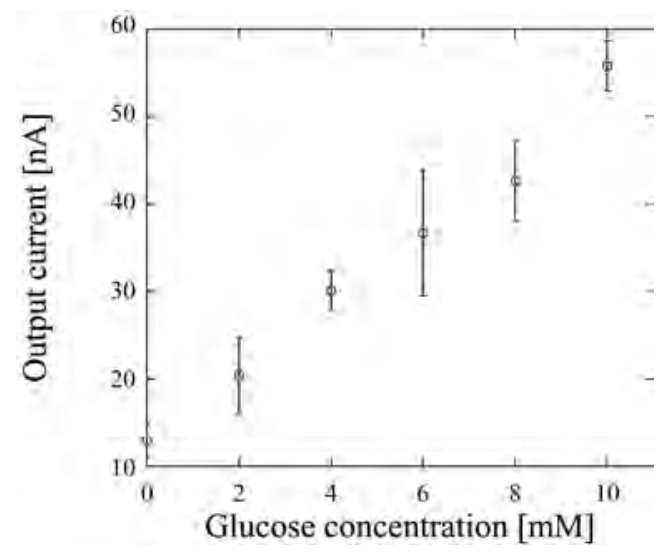

Fig. 8. Calibration characteristics for glucose detection using the E-LSPR biosensor.

\section{Conclusions}

We achieved the multiple detection of antigen-antibody and enzymatic reactions using the E-LSPR biosensor; this indicates the applicability of this system for diabetes diagnostics. The LSPR absorbance strength and electrochemical characteristics of the system were observed to change owing to the antigen-antibody and enzyme-substrate reactions. Furthermore, the glucose concentration was successfully determined on the basis of the principle of electrochemical detection. Thus, the E-LSPR biosensor has great potential for application in diabetes diagnostics. Because of its characteristics, this biosensor can be applied as a novel biosensor in a simple test kit in several fields such as medicine, food quality control, and environmental monitoring.

\section{Acknowledgments}

We thank Dr. Naoki Nagatani of the Okayama University of Science for preparing this manuscript. This study was supported by the System Development Program for Advanced Measurement and Analysis (SENTAN) of the Japan Science and Technology Agency (JST).

\section{References}

1 T. Vo-Dinh, B. M. Cullum and D. L. Stokes: Sens. Actuators, B 74 (2001) 2.

2 A. Leung, P. M. Shankar and R. Mutharasan: Sens. Actuators, B 125 (2007) 688.

3 J. J. Gooding: Anal. Chim. Acta 559 (2006) 137.

4 H. Kudo, T. Sawada, E. Kazawa, H. Yoshida, Y. Iwasaki and K. Mitsubayashi: Biosens. Bioelectron. 22 (2006) 558.

5 J. Pepper, R. Noring, M. Klempner, B. Cunningham, A. Petrovich, R. Bousquet, C. Clapp, J. Brady and B. Hugh: Sens. Actuators, B 96 (2003) 565. 
6 A. Poscia, M. Mascini, D. Moscone, M. Luzzana, G. Caramenti, P. Cremonesi, F. Valgimigli, C. Bongiovanni and M. Varalli: Biosens. Bioelectron. 18 (2003) 891.

7 R. Nagata, S. A. Clark, K. Yokoyama, E. Tamiya and I. Karube: Anal. Chim. Acta 304 (1995) 157.

8 T. S. Ahmadi, S. L. Logunov and M. A. El-Sayed: Clusters, Composites, and Thin Films, Nanostructured Materials, eds. V. M. Shalaev and M. Moskovits (American Chemical Society, Washington D. C., 1997) p. 125.

9 C. Sönnichsen, B. M. Reinhard, J. Liphardt and A. P. Alivisatos: Nat. Biotechnol. 23 (2005) 741.

10 A. J. Haes and R. P. Van Duyne: J. Am. Chem. Soc. 124 (2002) 10596.

11 D. A. Stuart, C. R. Yonzon, X. Zhang, O. Lyandres, N. C. Shah, M. R. Glucksberg, J. T. Walsh and R. P. Van Duyne: Anal. Chem. 77 (2005) 4013.

12 N. Nath and A. Chilkoti: Anal. Chem. 74 (2002) 504.

13 T. Endo, R. Ikeda, Y. Yanagida and T. Hatsuzawa: Anal. Chim. Acta 611 (2008) 205.

14 F. Frederix, J. M. Friedt, K. H. Choi, W. Laureyn, A. Campitelli, D. Mondelaers, G. Maes and G. Borghs: Anal. Chem. 75 (2003) 6894.

15 T. Endo, S. Yamamura, N. Nagatani, Y. Morita, Y. Takamura and E. Tamiya: Sci. Tech. Adv. Mater. 6 (2005) 491.

16 T. Endo, K. Kerman, N. Nagatani, Y. Takamura and E. Tamiya: Anal. Chem. 77 (2005) 6976.

17 T. Endo, K. Kerman, N. Nagatani, H. M. Hiep, D. K. Kim, Y. Yonezawa, K. Nakano and E. Tamiya: Anal. Chem. 78 (2006) 6465.

18 T. Endo, K. Kerman, N. Nagatani and E. Tamiya: J. Phys.: Condens. Matter 19 (2007) 215201.

19 T. Endo, S. Yamamura, K. Kerman and E. Tamiya: Anal. Chim. Acta 614 (2008) 182.

20 H. M. Hiep, T. Endo, M. Saito, M. Chikae, D. K. Kim, S. Yamamura, Y. Takamura and E. Tamiya: Anal. Chem. 80 (2008) 1859.

21 W. K. Ward, L. B. Jansen, E. Anderson, G. Reach, J. C. Klein and G. S. Wilson: Biosens. Bioelectron. 17 (2002) 181.

22 J. C. Pickup, F. Hussain, N. D. Evans and N. Sachedina: Biosens. Bioelectron. 20 (2005) 1897. 